MATHEMATICS OF COMPUTATION

Volume 81, Number 277, January 2012, Pages 219-231

S 0025-5718(2011)02514-7

Article electronically published on May 16, 2011

\title{
MAXIMUM PRINCIPLE AND CONVERGENCE OF CENTRAL SCHEMES BASED ON SLOPE LIMITERS
}

\author{
ORHAN MEHMETOGLU AND BOJAN POPOV
}

\begin{abstract}
A maximum principle and convergence of second order central schemes is proven for scalar conservation laws in dimension one. It is well known that to establish a maximum principle a nonlinear piecewise linear reconstruction is needed and a typical choice is the minmod limiter. Unfortunately, this implies that the scheme uses a first order reconstruction at local extrema. The novelty here is that we allow local nonlinear reconstructions which do not reduce to first order at local extrema and still prove maximum principle and convergence.
\end{abstract}

\section{INTRODUCTION}

This paper is concerned with scalar hyperbolic conservation laws

$$
\begin{cases}u_{t}+f(u)_{x}=0, & (x, t) \in \mathbb{R} \times(0, \infty) \\ u(x, 0)=u^{0}(x), & x \in \mathbb{R}\end{cases}
$$

where $f$ is a given flux function. In recent years there has been a lot of development in mathematical theory and construction of numerical algorithms for (1.1). Even though the existence-uniqueness theory is complete, there are many numerically efficient methods for which the questions of convergence and error estimates are still open. For example, there are many second or higher order nonoscillatory schemes based on minmod or other limiters which are numerically robust but theoretical results about convergence or error estimates are still missing [3, 4, 5, 6, 7, 8, A fundamental step in the design of such a numerical algorithm is a piecewise linear slope reconstruction; see for example [4, 8]. Nonlinear limiters such as minmod, generalized minmod, UNO, and superbee are widely used. A common shortcoming of all proofs is that the linear reconstruction used must reduce to first order (zero slope) at local extrema in order to prove a maximum principle for the scheme (see for example [8]), and when that limitation is not imposed there are typically no theoretical results for the methods even though they perform well in practice 3. 4, 8, 2].

In this paper we consider a class of nonlinear reconstructions which include and are motivated by the so-called minimum angle plane reconstruction (MAPR) introduced in [2]. The key idea (see section 2 here or [2]), is that at local extrema the

Received by the editor May 13, 2010 and, in revised form, September 9, 2010 and December $3,2010$.

2010 Mathematics Subject Classification. Primary 65M12; Secondary 65M08.

This material is based on work supported by the National Science Foundation grant DMS0811041. This publication is based on work partially supported by Award No. KUS-C1-016-04, made by King Abdullah University of Science and Technology (KAUST).

(C)2011 American Mathematical Society Reverts to public domain 28 years from publication 
slope of the reconstruction is not set to zero but it is limited by the smallest local slope. As a consequence, the maximum principle proofs for generalized minmod limiters in [8, 6] are no longer valid and a new approach is needed.

The paper is organized as follows. In section 2, we give a short overview of the Nessyahu-Tadmor (NT) staggered central scheme and prove a maximum principle when MAPR-like limiters are used. The result holds for a conservation law with a Lipschitz flux (see Theorem 2.2), and also for any smooth convex flux (see Theorem 2.5). In section 3, we prove that in the case of strictly convex flux the NT scheme satisfies a one-sided Lipschitz stability estimate and converges to the entropy solution when the MAPR-like limiter is properly selected. This is a generalization of the result in [10] for the minmod slope reconstruction.

\section{Nonoscillatory Central SCHEMes}

In this section we are going to consider second order nonoscillatory staggered central difference approximations to the scalar conservation law,

$$
u_{t}+f(u)_{x}=0
$$

We restrict our attention to the one-dimensional staggered NT scheme 8 , which was the motivation for the construction of many other central staggered schemes; see for example [6, 7, 2, 1]. Even though it may be possible to prove more general results, we will only consider the standard NT scheme setup. Let $v(x, t)$ be an approximate solution to (2.1), and assume that the space mesh $\Delta x$ and the time mesh $\Delta t$ are uniform. Let $x_{j}:=j \Delta x, j \in \mathbb{Z}, I_{j}:=\left\{s:\left|s-x_{j}\right|<\frac{\Delta x}{2}\right\}, t^{n}=n \Delta t$, $n \in \mathbb{N}$, and

$$
v_{j}^{n}:=\frac{1}{\Delta x} \int_{I_{j}} v\left(x, t^{n}\right) d x
$$

be the average of $v\left(x, t^{n}\right)$ over $I_{j}$. Next, assume that $v\left(\cdot, t^{n}\right)$ is a piecewise linear function of the form

$$
v\left(x, t^{n}\right)=\sum L_{j}\left(x, t^{n}\right):=\sum\left(v_{j}^{n}+\left(x-x_{j}\right) \frac{1}{\Delta x} v_{j}^{\prime}\right) \chi_{j}(x),
$$

where $\chi_{j}$ is the characteristic function over $I_{j}$ and $\frac{1}{\Delta x} v_{j}^{\prime}$ is the numerical derivative of $v\left(x=x_{j}, t^{n}\right)$, which is yet to be specified. We proceed by integrating (2.1) over $I_{j+\frac{1}{2}} \times\left(t^{n}, t^{n+1}\right)$ which yields

$$
\begin{aligned}
v_{j+\frac{1}{2}}\left(t^{n+1}\right) & =\frac{1}{\Delta x}\left(\int_{x_{j}}^{x_{j+\frac{1}{2}}} L_{j}\left(x, t^{n}\right) d x+\int_{x_{j+\frac{1}{2}}}^{x_{j+1}} L_{j+1}\left(x, t^{n}\right) d x\right) \\
& -\frac{1}{\Delta x}\left(\int_{t^{n}}^{t^{n+1}} f\left(v\left(x_{j+1}, \tau\right)\right) d \tau-\int_{t^{n}}^{t^{n+1}} f\left(v\left(x_{j}, \tau\right)\right) d \tau\right) .
\end{aligned}
$$

The first two linear integrands on the right of (2.4), $L_{j}\left(x, t^{n}\right)$ and $L_{j+1}\left(x, t^{n}\right)$, can be integrated exactly and if the CFL condition (with mesh ratio $\lambda:=\frac{\Delta t}{\Delta x}$ )

$$
\lambda \max _{x_{j} \leq x \leq x_{j+1}}\left|f^{\prime}(v(x, t))\right| \leq \frac{1}{2}, \quad j \in \mathbb{Z},
$$

is satisfied, then the last two integrands on the right of (2.4), $f\left(v\left(x_{j}, \tau\right)\right)$ and $f\left(v\left(x_{j+1}, \tau\right)\right)$, can be integrated approximately by the midpoint rule at the expense 
of the $O(\Delta t)^{3}$ local truncation error. Thus we arrive at

$$
v_{j+\frac{1}{2}}^{n+1}=\frac{1}{2}\left(v_{j}^{n}+v_{j+1}^{n}\right)+\frac{1}{8}\left(v_{j}^{\prime}-v_{j+1}^{\prime}\right)-\lambda\left(f\left(v_{j+1}^{n+\frac{1}{2}}\right)-f\left(v_{j}^{n+\frac{1}{2}}\right)\right) .
$$

The approximate value for the midpoint rule in time, $v_{j}^{n+\frac{1}{2}}$, satisfying second order accuracy requirement can be chosen as

$$
v_{j}^{n+\frac{1}{2}}=v_{j}^{n}-\frac{1}{2} \lambda f_{j}^{\prime},
$$

owing to Taylor expansion and (2.1). Here, $\frac{1}{\Delta x} f_{j}^{\prime}$ stands for an approximate numerical derivative of the flux $f\left(v\left(x=x_{j}, t^{n}\right)\right)$, which is yet to be determined. Although there are many different recipes to construct $v_{j}^{\prime}$ and $f_{j}^{\prime}$, in this paper the following are considered to be the approximations of the numerical derivatives

$$
\begin{gathered}
v_{j}^{\prime}=\mathrm{m}\left(v_{j+1}^{n}-v_{j}^{n}, v_{j}^{n}-v_{j-1}^{n}\right), \\
f_{j}^{\prime}=f^{\prime}\left(v_{j}^{n}\right) v_{j}^{\prime},
\end{gathered}
$$

where $\mathrm{m}(\cdot, \cdot)$ is the modified minmod limiter

$$
\mathrm{m}(a, b):= \begin{cases}\operatorname{sg}(a) \min (|a|,|b|), & a b \geq 0 \\ \sigma \min (|a|,|b|), & a b<0\end{cases}
$$

with $\sigma \in \mathbb{R},|\sigma| \leq 1$.

Remark 2.1. Note that the choice

$$
\sigma=\operatorname{sg}(s), \quad \text { where } \quad s= \begin{cases}a, & |a| \leq|b|, \\ b, & |b| \leq|a|,\end{cases}
$$

recovers the MAPR limiter introduced in [2].

Using the approximate slopes (2.8) and the approximate flux derivatives (2.9), we end up with a family of central differencing schemes in the predictor-corrector form

$$
\begin{gathered}
v_{j}^{n+\frac{1}{2}}=v_{j}^{n}-\frac{1}{2} \lambda f_{j}^{\prime}, \\
v_{j+\frac{1}{2}}^{n+1}=\frac{1}{2}\left(v_{j}^{n}+v_{j+1}^{n}\right)+\frac{1}{8}\left(v_{j}^{\prime}-v_{j+1}^{\prime}\right)-\lambda\left(f\left(v_{j+1}^{n+\frac{1}{2}}\right)-f\left(v_{j}^{n+\frac{1}{2}}\right)\right) .
\end{gathered}
$$

Note that (2.10) allows the predicted values $\left\{v_{j}^{n+\frac{1}{2}}\right\}_{j}$ (see (2.12)) to violate maximum principle. That is, the minimum/maximum of the sequence $\left\{v_{j}^{n+\frac{1}{2}}\right\}_{j}$ could be smaller/larger than that of $\left\{v_{j}^{n}\right\}_{j}$. This is going to be the main difficulty in proving maximum principle. We begin with a result in a simpler setting when the flux is globally Lipschitz continuous.

Theorem 2.2. Let $v_{j}^{\prime}$ be chosen by (2.8) and $f_{j}^{\prime}=f^{\prime}\left(v_{j}^{n}\right) v_{j}^{\prime}$. If $f$ is globally Lipschitz continuous, then the scheme described by (2.12)-(2.13) under the CFL condition

$$
\lambda\left\|f^{\prime}\right\|_{L^{\infty}(\mathbb{R})} \leq \beta \leq \frac{\sqrt{2}-1}{2}
$$

satisfies the maximum principle

$$
\min \left(v_{j}^{n}, v_{j+1}^{n}\right) \leq v_{j+\frac{1}{2}}^{n+1} \leq \max \left(v_{j}^{n}, v_{j+1}^{n}\right) .
$$


Proof. First, we rewrite the term $f\left(v_{j+1}^{n+\frac{1}{2}}\right)-f\left(v_{j}^{n+\frac{1}{2}}\right)$ in (2.13) as

$$
f\left(v_{j+1}^{n+\frac{1}{2}}\right)-f\left(v_{j}^{n+\frac{1}{2}}\right)=f^{\prime}\left(\xi_{j+\frac{1}{2}}^{n+\frac{1}{2}}\right)\left(v_{j+1}^{n+\frac{1}{2}}-v_{j}^{n+\frac{1}{2}}\right),
$$

where $\min \left(v_{j}^{n+\frac{1}{2}}, v_{j+1}^{n+\frac{1}{2}}\right) \leq \xi_{j+\frac{1}{2}}^{n+\frac{1}{2}} \leq \max \left(v_{j}^{n+\frac{1}{2}}, v_{j+1}^{n+\frac{1}{2}}\right)$. Observe that,

$$
\begin{aligned}
\left|v_{j+1}^{n+\frac{1}{2}}-v_{j}^{n+\frac{1}{2}}\right| & =\left|v_{j+1}^{n}-v_{j}^{n}-\frac{\lambda}{2}\left(f^{\prime}\left(v_{j+1}^{n}\right) v_{j+1}^{\prime}-f^{\prime}\left(v_{j}^{n}\right) v_{j}^{\prime}\right)\right| \\
& \leq\left(1+\frac{\lambda}{2}\left(\left|f^{\prime}\left(v_{j+1}^{n}\right)\right|+\left|f^{\prime}\left(v_{j}^{n}\right)\right|\right)\right)\left|v_{j+1}^{n}-v_{j}^{n}\right| \\
& \leq(1+\beta)\left|v_{j+1}^{n}-v_{j}^{n}\right| .
\end{aligned}
$$

Using (2.14), (2.16) and (2.17) we find an upper bound for (2.13),

$$
\begin{aligned}
v_{j+\frac{1}{2}}^{n+1} & \leq \frac{1}{2}\left(v_{j}^{n}+v_{j+1}^{n}\right)+\frac{1}{8}\left|v_{j}^{\prime}-v_{j+1}^{\prime}\right|+\lambda\left|f\left(v_{j+1}^{n+\frac{1}{2}}\right)-f\left(v_{j}^{n+\frac{1}{2}}\right)\right| \\
& \leq \frac{1}{2}\left(v_{j}^{n}+v_{j+1}^{n}\right)+\left(\frac{1}{2}+\beta\right)^{2}\left|v_{j+1}-v_{j}\right| \\
& \leq \frac{1}{2}\left(v_{j}^{n}+v_{j+1}^{n}\right)+\frac{1}{2}\left|v_{j+1}-v_{j}\right|=\max \left(v_{j}^{n}, v_{j+1}^{n}\right),
\end{aligned}
$$

and similarly a lower bound,

$$
\begin{aligned}
v_{j+\frac{1}{2}}^{n+1} & \geq \frac{1}{2}\left(v_{j}^{n}+v_{j+1}^{n}\right)-\frac{1}{8}\left|v_{j}^{\prime}-v_{j+1}^{\prime}\right|-\lambda\left|f\left(v_{j+1}^{n+\frac{1}{2}}\right)-f\left(v_{j}^{n+\frac{1}{2}}\right)\right| \\
& \geq \frac{1}{2}\left(v_{j}^{n}+v_{j+1}^{n}\right)-\left(\frac{1}{2}+\beta\right)^{2}\left|v_{j+1}-v_{j}\right| \\
& \geq \frac{1}{2}\left(v_{j}^{n}+v_{j+1}^{n}\right)-\frac{1}{2}\left|v_{j+1}-v_{j}\right|=\min \left(v_{j}^{n}, v_{j+1}^{n}\right) .
\end{aligned}
$$

The above two bounds prove the theorem.

Remark 2.3. This maximum principle proof implies the TVD bound for the NT scheme proven in Theorem 3.2 from [8]. The proof of the TVD bound in [8] is not valid for the Jacobian form which we use here (see (2.8)-(2.9) in this paper or (3.16a)-(3.16b) in $[8)$, even for the standard minmod limiter $(\sigma=0$ in (2.10) $)$. Hence, the present result extends Theorem 3.2 from 8 for the special choice of numerical derivative and numerical flux (2.8) $-(2.9)$ considered here.

Definition 2.4. The range of a function $g: \mathbb{R} \rightarrow \mathbb{R}$ is defined to be the interval

$$
R(g):=\left[\operatorname{essinf}_{x \in \mathbb{R}} g(x), \operatorname{esssup}_{x \in \mathbb{R}} g(x)\right] .
$$

Theorem 2.5. Let $v_{j}^{\prime}$ be chosen as in (2.8) and $f_{j}^{\prime}=f^{\prime}\left(v_{j}^{n}\right) v_{j}^{\prime}$. If $f$ is strictly convex, that is, there exists constants $\gamma_{1} \leq \gamma_{2}$ such that

$$
0<\gamma_{1} \leq f^{\prime \prime} \leq \gamma_{2},
$$

then the scheme described by (2.12), (2.13) satisfies the maximum principle (2.15) under the CFL condition

$$
\lambda \max _{w \in R\left(u^{0}\right)}\left|f^{\prime}(w)\right| \leq \beta,
$$

where $\beta$ is a fixed constant which depends only on $\gamma_{1}$ and $\gamma_{2}$; see (2.31). 
Proof. First, we observe

$$
\begin{aligned}
|f(b)-f(a)| & =\left|\int_{a}^{b} f^{\prime}(t) d t\right|=\left|\int_{a}^{b}\left(\int_{\frac{a+b}{2}}^{t} f^{\prime \prime}(s) d s+f^{\prime}\left(\frac{a+b}{2}\right)\right) d t\right| \\
& \leq\left(\frac{\gamma_{2}}{4}|b-a|+\left|f^{\prime}\left(\frac{a+b}{2}\right)\right|\right)|b-a| .
\end{aligned}
$$

Let $a=v_{j}^{n+\frac{1}{2}}$ and $b=v_{j+1}^{n+\frac{1}{2}}$ in the above inequality, then

$$
\begin{aligned}
& \left|f\left(v_{j+1}^{n+\frac{1}{2}}\right)-f\left(v_{j}^{n+\frac{1}{2}}\right)\right| \\
& \quad \leq\left(\frac{\gamma_{2}}{4}\left|v_{j+1}^{n+\frac{1}{2}}-v_{j}^{n+\frac{1}{2}}\right|+\left|f^{\prime}\left(\frac{v_{j}^{n+\frac{1}{2}}+v_{j+1}^{n+\frac{1}{2}}}{2}\right)\right|\right)\left|v_{j+1}^{n+\frac{1}{2}}-v_{j}^{n+\frac{1}{2}}\right| .
\end{aligned}
$$

Next, we bound the terms appearing on the right-hand side. We start with

$$
\begin{aligned}
\left|v_{j+1}^{n+\frac{1}{2}}-v_{j}^{n+\frac{1}{2}}\right| & =\left|v_{j+1}^{n}-v_{j}^{n}-\frac{\lambda}{2}\left(f^{\prime}\left(v_{j+1}^{n}\right) v_{j+1}^{\prime}-f^{\prime}\left(v_{j}^{n}\right) v_{j}^{\prime}\right)\right| \\
& \leq\left(1+\frac{\lambda}{2}\left(\left|f^{\prime}\left(v_{j+1}^{n}\right)\right|+\left|f^{\prime}\left(v_{j}^{n}\right)\right|\right)\right)\left|v_{j+1}^{n}-v_{j}^{n}\right| \\
& \leq(1+\beta)\left|v_{j+1}^{n}-v_{j}^{n}\right| \leq \frac{1+\beta}{\gamma_{1}}\left|f^{\prime}\left(v_{j+1}^{n}\right)-f^{\prime}\left(v_{j}^{n}\right)\right| \\
& \leq \frac{2(1+\beta)}{\gamma_{1}} \max _{j}\left|f^{\prime}\left(v_{j}^{n}\right)\right| .
\end{aligned}
$$

Note that the inequality

$$
\left|\frac{\lambda}{4}\left(f^{\prime}\left(v_{j}^{n}\right) v_{j}^{\prime}+f^{\prime}\left(v_{j+1}^{n}\right) v_{j+1}^{\prime}\right)\right| \leq \frac{\beta}{2}\left|v_{j+1}^{n}-v_{j}^{n}\right|
$$

implies

$$
\min \left(v_{j}^{n}, v_{j+1}^{n}\right) \leq \frac{v_{j}^{n+\frac{1}{2}}+v_{j+1}^{n+\frac{1}{2}}}{2} \leq \max \left(v_{j}^{n}, v_{j+1}^{n}\right)
$$

for all $\beta \leq 1$. From (2.20) and (2.26) it follows that

$$
\left|f^{\prime}\left(\frac{v_{j+1}^{n+\frac{1}{2}}+v_{j}^{n+\frac{1}{2}}}{2}\right)\right| \leq \max _{j}\left|f^{\prime}\left(v_{j}^{n}\right)\right| .
$$

We use (2.23), (2.24) and (2.27) and derive

$$
\begin{aligned}
\left|f\left(v_{j+1}^{n+\frac{1}{2}}\right)-f\left(v_{j}^{n+\frac{1}{2}}\right)\right| & \leq\left(\frac{\gamma_{2}(1+\beta)}{2 \gamma_{1}}+1\right) \max _{j}\left|f^{\prime}\left(v_{j}^{n}\right)\right|\left|v_{j+1}^{n+\frac{1}{2}}-v_{j}^{n+\frac{1}{2}}\right| \\
& \leq\left(\frac{\gamma_{2}(1+\beta)}{2 \gamma_{1}}+1\right)(1+\beta) \max _{j}\left|f^{\prime}\left(v_{j}^{n}\right)\right|\left|v_{j+1}^{n}-v_{j}^{n}\right| .
\end{aligned}
$$

Using (2.28) in (2.13) gives the estimates

$$
\begin{aligned}
v_{j+\frac{1}{2}}^{n+1} & \leq \frac{1}{2}\left(v_{j}^{n}+v_{j+1}^{n}\right)+\frac{1}{8}\left|v_{j}^{\prime}-v_{j+1}^{\prime}\right|+\lambda\left|f\left(v_{j+1}^{n+\frac{1}{2}}\right)-f\left(v_{j}^{n+\frac{1}{2}}\right)\right| \\
& \leq \frac{1}{2}\left(v_{j}^{n}+v_{j+1}^{n}\right)+\left(\frac{1}{4}+\beta(1+\beta)\left(\frac{\gamma_{2}(1+\beta)}{2 \gamma_{1}}+1\right)\right)\left|\delta_{j+\frac{1}{2}}^{n}\right|
\end{aligned}
$$


and

$$
\begin{aligned}
v_{j+\frac{1}{2}}^{n+1} & \geq \frac{1}{2}\left(v_{j}^{n}+v_{j+1}^{n}\right)-\frac{1}{8}\left|v_{j}^{\prime}-v_{j+1}^{\prime}\right|-\lambda\left|f\left(v_{j+1}^{n+\frac{1}{2}}\right)-f\left(v_{j}^{n+\frac{1}{2}}\right)\right| \\
& \geq \frac{1}{2}\left(v_{j}^{n}+v_{j+1}^{n}\right)-\left(\frac{1}{4}+\beta(1+\beta)\left(\frac{\gamma_{2}(1+\beta)}{2 \gamma_{1}}+1\right)\right)\left|\delta_{j+\frac{1}{2}}^{n}\right|,
\end{aligned}
$$

where $\delta_{j+\frac{1}{2}}^{n}=v_{j+1}^{n}-v_{j}^{n}$. Hence, under the CFL condition

$$
\beta(1+\beta)\left(\frac{\gamma_{2}(1+\beta)}{2 \gamma_{1}}+1\right) \leq \frac{1}{4},
$$

we have

$$
\min \left(v_{j}^{n}, v_{j+1}^{n}\right) \leq v_{j+\frac{1}{2}}^{n+1} \leq \max \left(v_{j}^{n}, v_{j+1}^{n}\right) .
$$

Remark 2.6. In the case of Burgers' equation (2.31) holds when $\beta \leq 0.1397$ which implies that the CFL condition (2.21) is satisfied with $\beta=0.1397$.

Remark 2.7. It is possible to prove a result similar to Theorem 2.5 for nonconvex fluxes which satisfy

$$
0<\gamma_{1} \leq f^{(k)}(x) \leq \gamma_{2} .
$$

Such functions are called $k$-monotone functions and any fixed polynomial is a $k$ monotone function for some $k$. The proof of this result will be given elsewhere.

\section{ONE-SIDED $l_{2}$ STABILITY AND CONVERGENCE}

Recall that $\left\{v_{j}^{n}\right\}_{j \in \mathbb{Z}}$ and $\left\{v_{j+\frac{1}{2}}^{n+1}\right\}_{j \in \mathbb{Z}}$ are the sequences of cell averages of the numerical solution of NT scheme at time $t^{n}$ and $t^{n+1}$, respectively. Let us introduce the following notation for the jump sequences of the NT solution

$$
\delta_{j+\frac{1}{2}}:=v_{j+1}^{n}-v_{j}^{n} \text { and } \delta_{j}:=v_{j+\frac{1}{2}}^{n+1}-v_{j-\frac{1}{2}}^{n+1}, \quad j \in \mathbb{Z},
$$

at times $t^{n}$ and $t^{n+1}$, respectively. With this notation we have the following theorem which is the main result of this section.

Theorem 3.1. Let $u^{0} \in L^{\infty}(\mathbb{R})$, let $f$ be strictly convex in $R\left(u^{0}\right)$ and let $f^{\prime \prime}$ be bounded on $\mathbb{R}$. That is, there exist constants $\gamma_{1}$ and $\gamma_{2}$ such that

$$
\begin{gathered}
0<\gamma_{1} \leq f^{\prime \prime}(w), \quad \forall w \in R\left(u^{o}\right), \\
f^{\prime \prime}(x) \leq \gamma_{2}, \quad \forall x \in \mathbb{R} .
\end{gathered}
$$

Then there exists a constant $\beta$ which depends only on the ratio $\gamma_{1} / \gamma_{2}$ such that under the CFL condition

$$
\lambda \max _{w \in R\left(u^{0}\right)}\left|f^{\prime}(w)\right| \leq \beta,
$$

the NT scheme with the limiter (2.10) and $0 \leq \sigma \leq 1$ satisfies the following onesided Lipschitz condition

$$
\left\|\left\{\left(\delta_{j}\right)_{+}\right\}_{j \in \mathbb{Z}}\right\|_{l_{2}} \leq\left\|\left\{\left(\delta_{j+\frac{1}{2}}\right)_{+}\right\}_{j \in \mathbb{Z}}\right\|_{l_{2}},
$$

where we use the standard "+" notation: $x_{+}=\max (x, 0)$. In other words, the $l_{2}$ norm of the positive jumps does not increase in time. 
Remark 3.2. Taking $\sigma=0$ in (2.10), we obtain the original minmod limiter. It is easy to see that among all $\sigma,|\sigma| \leq 1$, the choice $\sigma=1$ in (2.10) minimizes the size of the positive jumps in the piecewise linear numerical solution. These are the so-called entropy violating jumps for convex flux (see [9] and Remark 1 on page 422 in [8]), and one needs to have control of their size in order to prove convergence to the entropy solution.

Proof. It is an easy exercise in real analysis to show that every bounded sequence can be decomposed into a union of monotone subsequences. That is, given the sequence $\left\{v_{j}^{n}\right\}_{j \in \mathbb{Z}}$ there exists a nonempty collection of index sets $\Lambda_{k}:=\left\{j \mid j_{\min }^{k} \leq\right.$ $\left.j \leq j_{\max }^{k}\right\}$ such that $j_{\max }^{k}=j_{\min }^{k+1}$ for all $k \in \mathbb{Z}$, and $\left\{v_{j}^{n}\right\}_{j \in \Lambda_{k}}$ is nondecreasing if $k$ is even, and nonincreasing if $k$ is odd. This decomposition is not necessarily unique. To fix one, we choose $\left\{\Lambda_{k}\right\}_{k}$ such that $\Lambda_{k}$ has maximum number of terms for each even $k$. That is, for all even $k$ such that $\Lambda_{k}$ is nonempty, we have

$$
\begin{aligned}
& v_{j_{\min }^{k}-1}>v_{j_{\min }^{k}}, \\
& v_{j_{\max }^{k}+1}>v_{j_{\max }^{k}} .
\end{aligned}
$$

Note that we have a single set $\Lambda_{k}$ if the data is monotone and that (3.5) and (3.6) only make sense if $j_{\min }^{k}>-\infty$ and $j_{\max }^{k}<\infty$, respectively. With this notation, there are only two possibilities to generate nonnegative jumps $\delta_{j}$ in the new sequence $\left\{v_{j+1 / 2}^{n+1}\right\}_{j \in \mathbb{Z}}$ starting from the old sequence $\left\{v_{j}^{n}\right\}_{j \in \mathbb{Z}}$ :

(i) If $v_{j-1}^{n} \leq v_{j}^{n} \leq v_{j+1}^{n}$, i.e. $j-1, j, j+1 \in \Lambda_{k}$ for some even $k$, then we have an internal jump, i.e., generated from the interior of a nondecreasing monotone subsequence $\Lambda_{k}$.

(ii) If $\left(v_{j}^{n}-v_{j-1}^{n}\right)\left(v_{j+1}^{n}-v_{j}^{n}\right) \leq 0$ and at least one of these jumps $\left(\delta_{j-\frac{1}{2}}\right.$ or $\left.\delta_{j+\frac{1}{2}}\right)$ is not zero. That is, $j-1, j \in \Lambda_{k}$ and $j, j+1 \in \Lambda_{k+1}$ for some $k$, then we have a boundary jump, i.e., generated on the boundary of $\Lambda_{k}$ and $\Lambda_{k+1}$.

The jumps generated in case 1 are always nonnegative, whereas the jumps generated in case 2 may have different signs.

Next, without loss of generality, we assume that there is at least one nondecreasing subsequence of $\left\{v_{j}^{n}\right\}_{j \in \mathbb{Z}}$, say with index set $\Lambda_{0}=\{0, \ldots, m\}$. Otherwise, there is nothing to prove. We define the modified cell averages $\left\{\bar{v}_{j}^{n}\right\}_{j \in \mathbb{Z}}$, as

$$
\bar{v}_{j}^{n}:= \begin{cases}v_{0}^{n}, & j \leq 0, \\ v_{j}^{n}, & 0<j<m, \\ v_{m}^{n}, & m \leq j,\end{cases}
$$

which is the so-called constant extension of $\left\{v_{j}^{n}\right\}_{j=0}^{m}$; see [10]. The jumps of the modified cell averages are given by

$$
\bar{\delta}_{j+\frac{1}{2}}:=\bar{v}_{j+1}^{n}-\bar{v}_{j}^{n} \quad \text { and } \quad \bar{\delta}_{j}:=\bar{v}_{j+\frac{1}{2}}^{n+1}-\bar{v}_{j-\frac{1}{2}}^{n+1}, \quad j \in \mathbb{Z} .
$$

The following facts follow from the definition of $\left\{\bar{v}_{j}^{n}\right\}_{j \in \mathbb{Z}}$ :

$$
\begin{aligned}
& \bar{v}_{j+\frac{1}{2}}^{n+1}=v_{0}^{n}, \quad j \leq-1, \\
& \bar{v}_{j+\frac{1}{2}}^{n+1}=v_{m}^{n}, j \geq m, \\
& \bar{v}_{j+\frac{1}{2}}=v_{j+\frac{1}{2}}, 1 \leq j \leq m-2 .
\end{aligned}
$$


Using the above we get

$$
\begin{aligned}
\bar{\delta}_{j} & =\left(\ldots, 0, \bar{\delta}_{0}, \bar{\delta}_{1}, \delta_{2}, \ldots, \delta_{m-2}, \bar{\delta}_{m-1}, \bar{\delta}_{m}, 0, \ldots\right), \\
\bar{\delta}_{j+\frac{1}{2}} & =\left(\ldots, 0, \delta_{\frac{1}{2}}, \delta_{\frac{3}{2}}, \ldots, \delta_{m-\frac{1}{2}}, 0, \ldots\right),
\end{aligned}
$$

with the convention that we drop any terms that do not make sense when $m \leq 3$. In view of (ii) and (iii) the nonnegative jumps of the new sequence can be decomposed into (interior and boundary) jumps generated by each $\Lambda_{k}$, for even $k$. Therefore, to prove the theorem it is enough to show that

$$
\left\|\left\{\left(\delta_{j}\right)_{+}\right\}_{j=0}^{m}\right\|_{l_{2}} \leq\left\|\left\{\left(\delta_{j+\frac{1}{2}}\right)_{+}\right\}_{j=0}^{m-1}\right\|_{l_{2}},
$$

because the left-hand side includes all nonnegative jumps that may be generated by $\Lambda_{0}$. For the sequence (3.7) the limiter (2.10) coincides with the minmod limiter. Hence, we can apply the one-sided stability result from [10] for a single nondecreasing sequence

$$
\sum_{j=0}^{m}\left(\bar{\delta}_{j}\right)^{2} \leq \sum_{j=0}^{m-1}\left(\delta_{j+\frac{1}{2}}\right)^{2} .
$$

Having (3.12) it suffices to show that

$$
\sum_{j=0}^{m}\left(\delta_{j}\right)_{+}^{2} \leq \sum_{j=0}^{m}\left(\bar{\delta}_{j}\right)^{2}
$$

We split the proof of (3.13) into four cases.

Case $1 . m \geq 4$.

We need the following lemma.

Lemma 3.3. The following inequality holds when $\sigma \geq 0$ in (2.10):

$$
\left(\delta_{1}\right)_{+}^{2}+\left(\delta_{0}\right)_{+}^{2} \leq\left(\bar{\delta}_{1}\right)^{2}+\left(\bar{\delta}_{0}\right)^{2} .
$$

Proof. We are going to prove the argument in two steps.

Step 1. First, we will show that $\left(\delta_{1}\right)^{2}-\left(\bar{\delta}_{1}\right)^{2} \leq 0$.

Observe that

$$
\begin{aligned}
\left(\delta_{1}\right)^{2}-\left(\bar{\delta}_{1}\right)^{2}=\left(\delta_{1}+\bar{\delta}_{1}\right)\left(\delta_{1}-\bar{\delta}_{1}\right) & =k_{1}^{n+1}\left(\left(v_{\frac{3}{2}}^{n+1}-v_{\frac{1}{2}}^{n+1}\right)-\left(\bar{v}_{\frac{3}{2}}^{n+1}-\bar{v}_{\frac{1}{2}}^{n+1}\right)\right) \\
& =k_{1}^{n+1}\left(\bar{v}_{\frac{1}{2}}^{n+1}-v_{\frac{1}{2}}^{n+1}\right),
\end{aligned}
$$

where $k_{1}^{n+1}:=\delta_{1}+\bar{\delta}_{1}>0$ as $\delta_{1}$ and $\bar{\delta}_{1}$ are both positive. Next, we need to check the sign of $\bar{v}_{\frac{1}{2}}^{n+1}-v_{\frac{1}{2}}^{n+1}$, where

$$
\begin{aligned}
& \bar{v}_{\frac{1}{2}}^{n+1}=\frac{1}{2}\left(\bar{v}_{0}^{n}+\bar{v}_{1}^{n}\right)+\frac{1}{8}\left(\bar{v}_{0}^{\prime}-\bar{v}_{1}^{\prime}\right)-\lambda\left(f\left(\bar{v}_{1}^{n+\frac{1}{2}}\right)-f\left(\bar{v}_{0}^{n+\frac{1}{2}}\right)\right), \\
& v_{\frac{1}{2}}^{n+1}=\frac{1}{2}\left(v_{0}^{n}+v_{1}^{n}\right)+\frac{1}{8}\left(v_{0}^{\prime}-v_{1}^{\prime}\right)-\lambda\left(f\left(v_{1}^{n+\frac{1}{2}}\right)-f\left(v_{0}^{n+\frac{1}{2}}\right)\right) .
\end{aligned}
$$

Note that $v_{1}^{\prime}=\mathrm{m}\left(v_{1}^{n}-v_{0}^{n}, v_{2}^{n}-v_{1}^{n}\right)=\bar{v}_{1}^{\prime}$, and $v_{1}^{n+\frac{1}{2}}=v_{1}^{n}-\frac{\lambda}{2} f^{\prime}\left(v_{1}^{n}\right) v_{1}^{\prime}=\bar{v}_{1}^{n+\frac{1}{2}}$, which follows from (3.9). Also observe that $\bar{v}_{0}^{\prime}=\mathrm{m}\left(0, v_{1}^{n}-v_{0}^{n}\right)=0$ and $v_{0}^{\prime} \geq 0$ 
since $\sigma \geq 0$ in (2.10). Subtract (3.17) from (3.16) to get

$$
\begin{aligned}
\bar{v}_{\frac{1}{2}}^{n+1}-v_{\frac{1}{2}}^{n+1} & =\frac{1}{8}\left(\bar{v}_{0}^{\prime}-v_{0}^{\prime}\right)+\lambda\left(f\left(\bar{v}_{0}^{n+\frac{1}{2}}\right)-f\left(v_{0}^{n+\frac{1}{2}}\right)\right) \\
& =-\frac{1}{8} v_{0}^{\prime}+\lambda\left(f\left(v_{0}^{n}\right)-f\left(v_{0}^{n+\frac{1}{2}}\right)\right) \\
& \leq-\frac{1}{8} v_{0}^{\prime}+\frac{\lambda^{2}}{2}\left|f^{\prime}\left(v_{0}^{n}\right)\right| v_{0}^{\prime} \max \left(\left|f^{\prime}\left(v_{0}^{n}\right)\right|,\left|f^{\prime}\left(v_{0}^{n}-\frac{\lambda}{2} f^{\prime}\left(v_{0}^{n}\right) v_{0}^{\prime}\right)\right|\right) .
\end{aligned}
$$

There are two possibilities:

(I) Suppose $\max \left(\left|f^{\prime}\left(v_{0}^{n}\right)\right|,\left|f^{\prime}\left(v_{0}^{n}-\frac{\lambda}{2} f^{\prime}\left(v_{o}^{n}\right) v_{0}^{\prime}\right)\right|\right)=\left|f^{\prime}\left(v_{0}^{n}\right)\right|$. Then we rewrite (3.18) as

$$
\begin{aligned}
\bar{v}_{\frac{1}{2}}^{n+1}-v_{\frac{1}{2}}^{n+1} & \leq-\frac{1}{8} v_{0}^{\prime}+\frac{1}{2}\left|\lambda f^{\prime}\left(v_{0}^{n}\right)\right|^{2} v_{0}^{\prime} \\
& \leq\left(-\frac{1}{8}+\frac{\beta^{2}}{2}\right) v_{0}^{\prime} \leq 0 .
\end{aligned}
$$

(II) Suppose $\max \left(\left|f^{\prime}\left(v_{0}^{n}\right)\right|,\left|f^{\prime}\left(v_{0}^{n}-\frac{\lambda}{2} f^{\prime}\left(v_{0}^{n}\right) v_{0}^{\prime}\right)\right|\right)=\left|f^{\prime}\left(v_{0}^{n}-\frac{\lambda}{2} f^{\prime}\left(v_{0}^{n}\right) v_{0}^{\prime}\right)\right|$. Then by the mean value theorem and (3.2) we have

$$
\left|v_{1}^{n}-v_{0}^{n}\right| \leq \frac{2}{\gamma_{1}} \max \left(\left|f^{\prime}\left(v_{1}^{n}\right)\right|,\left|f^{\prime}\left(v_{0}^{n}\right)\right|\right) .
$$

Now, by the above inequality, Taylor expansion, and (3.3) we have

$$
\begin{aligned}
\left|f^{\prime}\left(v_{0}^{n}-\frac{\lambda}{2} f^{\prime}\left(v_{0}^{n}\right) v_{0}^{\prime}\right)\right| & \leq\left|f^{\prime}\left(v_{0}^{n}\right)\right|\left(1+\frac{\lambda}{2} \gamma_{2} v_{0}^{\prime}\right) \\
& \leq\left|f^{\prime}\left(v_{0}^{n}\right)\right|\left(1+\lambda \frac{\gamma_{2}}{\gamma_{1}} \max \left(\left|f^{\prime}\left(v_{1}^{n}\right)\right|,\left|f^{\prime}\left(v_{0}^{n}\right)\right|\right)\right. \\
& \leq\left|f^{\prime}\left(v_{0}^{n}\right)\right|\left(1+\beta \frac{\gamma_{2}}{\gamma_{1}}\right) .
\end{aligned}
$$

We use this result in (3.18) to get

$$
\begin{aligned}
\bar{v}_{\frac{1}{2}}^{n+1}-v_{\frac{1}{2}}^{n+1} & \leq-\frac{1}{8} v_{0}^{\prime}+\frac{\lambda^{2}}{2}\left|f^{\prime}\left(v_{0}^{n}-\frac{\lambda}{2} f^{\prime}\left(v_{0}^{n}\right) v_{0}^{\prime}\right)\right|\left|f^{\prime}\left(v_{0}^{n}\right)\right| v_{0}^{\prime} \\
& \leq-\frac{1}{8} v_{0}^{\prime}+\frac{\lambda^{2}}{2}\left(1+\beta \frac{\gamma_{2}}{\gamma_{1}}\right)\left|f^{\prime}\left(v_{0}^{n}\right)\right|^{2} v_{0}^{\prime} \\
& \leq\left(-\frac{1}{8}+\frac{\beta^{2}}{2}\left(1+\beta \frac{\gamma_{2}}{\gamma_{1}}\right)\right) v_{0}^{\prime} \leq 0 .
\end{aligned}
$$

Therefore, in both cases (II) and (III), we conclude that

$$
\bar{v}_{\frac{1}{2}}^{n+1}-v_{\frac{1}{2}}^{n+1} \leq 0 .
$$

We finish the proof of Step 1 by observing that (3.15) and (3.23) imply

$$
\left(\delta_{1}\right)^{2}-\left(\bar{\delta}_{1}\right)^{2} \leq 0 .
$$

Step 2. We now prove that $\left(\delta_{0}\right)_{+}^{2}-\left(\bar{\delta}_{0}\right)^{2} \leq 0$. 
If $\delta_{0}<0$, then $\left(\delta_{0}\right)_{+}=0$ and there is nothing to prove. If $\delta_{0}>0$, we proceed as in the proof of Step 1 as follows,

$$
\begin{aligned}
\left(\delta_{0}\right)^{2}-\left(\bar{\delta}_{0}\right)^{2} & =\left(\delta_{0}+\bar{\delta}_{0}\right)\left(\delta_{0}-\bar{\delta}_{0}\right)=k_{0}^{n+1}\left(\left(v_{\frac{1}{2}}^{n+1}-v_{-\frac{1}{2}}^{n+1}\right)-\left(\bar{v}_{\frac{1}{2}}^{n+1}-\bar{v}_{-\frac{1}{2}}^{n+1}\right)\right) \\
& =k_{0}^{n+1}\left(\left(v_{\frac{1}{2}}^{n+1}-\bar{v}_{\frac{1}{2}}^{n+1}\right)+\left(v_{0}^{n}-v_{-\frac{1}{2}}^{n+1}\right)\right),
\end{aligned}
$$

where $k_{0}^{n+1}:=\delta_{0}+\bar{\delta}_{0}>0$. We still need to check the sign of $\delta_{0}-\bar{\delta}_{0}$ so we rewrite (2.30) for $v_{-\frac{1}{2}}^{n+1}$ and subtract it from $v_{0}^{n}$ to get

$$
\begin{aligned}
v_{0}^{n}-v_{-\frac{1}{2}}^{n+1} & \leq \frac{1}{2}\left(v_{0}^{n}-v_{-1}^{n}\right)+\left(\frac{1}{4}+\beta(1+\beta)\left(\frac{\gamma_{2}(1+\beta)}{2 \gamma_{1}}+1\right)\right)\left|v_{0}^{n}-v_{-1}^{n}\right| \\
& \leq\left(\frac{1}{4}-\beta(1+\beta)\left(\frac{\gamma_{2}(1+\beta)}{2 \gamma_{1}}+1\right)\right)\left(v_{0}^{n}-v_{-1}^{n}\right) .
\end{aligned}
$$

By similar arguments used in (3.19) and (3.22), we have

$$
v_{\frac{1}{2}}^{n+1}-\bar{v}_{\frac{1}{2}}^{n+1} \leq\left(\frac{1}{8}+\frac{\beta^{2}}{2} c\right) v_{0}^{\prime}
$$

where $c=1$ if the assumption of Case 1 holds, or $c=\left(1+\beta \gamma_{2} / \gamma_{1}\right)$ if the assumption of Case 2 holds. Now we apply these bounds to $\delta_{0}-\bar{\delta}_{0}$ :

$$
\begin{aligned}
\delta_{0}-\bar{\delta}_{0} & =\left(\left(v_{\frac{1}{2}}^{n+1}-\bar{v}_{\frac{1}{2}}^{n+1}\right)+\left(v_{0}^{n}-v_{-\frac{1}{2}}^{n+1}\right)\right) \\
& \leq\left(\frac{1}{8}+\frac{\beta^{2}}{2} c\right) v_{0}^{\prime}+\left(\frac{1}{4}-\beta(1+\beta)\left(\frac{\gamma_{2}(1+\beta)}{2 \gamma_{1}}+1\right)\right)\left(v_{0}^{n}-v_{-1}^{n}\right) \\
& \leq\left(\frac{1}{8}-\frac{\beta^{2}}{2} c-\beta(1+\beta)\left(\frac{\gamma_{2}(1+\beta)}{2 \gamma_{1}}+1\right)\right)\left(v_{0}^{n}-v_{-1}^{n}\right) \leq 0 .
\end{aligned}
$$

Finally, (3.24) and (3.27) imply

$$
\left(\delta_{0}\right)_{+}^{2}-\left(\bar{\delta}_{0}\right)^{2} \leq 0 .
$$

This proves Step 2 and Lemma 3.3

By symmetric arguments it can also be shown that $\left(\delta_{m-1}\right)_{+}^{2}-\left(\bar{\delta}_{m-1}\right)^{2} \leq 0$ and $\left(\delta_{m}\right)_{+}^{2}-\left(\bar{\delta}_{m}\right)^{2} \leq 0$. Gathering all the results we have established so far, we end up with

$$
\left(\delta_{0}\right)_{+}^{2}+\left(\delta_{1}\right)_{+}^{2}+\left(\delta_{m-1}\right)_{+}^{2}+\left(\delta_{m}\right)_{+}^{2} \leq\left(\bar{\delta}_{0}\right)_{+}^{2}+\left(\bar{\delta}_{1}\right)_{+}^{2}+\left(\bar{\delta}_{m-1}\right)_{+}^{2}+\left(\bar{\delta}_{m}\right)_{+}^{2},
$$

which leads us to

$$
\begin{aligned}
\sum_{j=0}^{m}\left(\delta_{j}\right)_{+}^{2} & =\left(\delta_{0}\right)_{+}^{2}+\left(\delta_{1}\right)_{+}^{2}+\left(\delta_{m-1}\right)_{+}^{2}+\left(\delta_{m}\right)_{+}^{2}+\sum_{j=2}^{m-2}\left(\delta_{j}\right)_{+}^{2} \\
& \leq\left(\bar{\delta}_{0}\right)_{+}^{2}+\left(\bar{\delta}_{1}\right)_{+}^{2}+\left(\bar{\delta}_{m-1}\right)_{+}^{2}+\left(\bar{\delta}_{m}\right)_{+}^{2}+\sum_{j=2}^{m-2}\left(\bar{\delta}_{j}\right)_{+}^{2} \\
& =\sum_{j=0}^{m}\left(\bar{\delta}_{j}\right)^{2} .
\end{aligned}
$$

This completes the proof for $m \geq 4$. Next, we consider the remaining cases.

Case 2. $m=3$. 
It is the same as Case 1 except that there are no middle jumps $\left(\delta_{2}, \ldots, \delta_{m-2}\right)$ in (3.10). Hence, we have

$$
\left(\delta_{0}\right)_{+}^{2}+\left(\delta_{1}\right)_{+}^{2}+\left(\delta_{2}\right)_{+}^{2}+\left(\delta_{3}\right)_{+}^{2} \leq\left(\bar{\delta}_{0}\right)^{2}+\left(\bar{\delta}_{1}\right)^{2}+\left(\bar{\delta}_{2}\right)^{2}+\left(\bar{\delta}_{3}\right)^{2} .
$$

Case 3. $m=2$.

We have already proved that

$$
\left(\delta_{0}\right)_{+}^{2}-\left(\bar{\delta}_{0}\right)^{2} \leq 0 .
$$

By symmetric arguments it is also true that

$$
\left(\delta_{2}\right)_{+}^{2}-\left(\bar{\delta}_{2}\right)^{2} \leq 0 .
$$

Next, we need to show that $\delta_{1} \leq \bar{\delta}_{1}$ since both are positive. From (3.18) we have $\bar{v}_{\frac{1}{2}}^{n+1} \leq v_{\frac{1}{2}}^{n+1}$ and by analogous arguments $\bar{v}_{\frac{3}{2}}^{n+1} \geq v_{\frac{3}{2}}^{n+1}$. Together they imply

$$
\delta_{1}=v_{\frac{3}{2}}^{n+1}-v_{\frac{1}{2}}^{n+1} \leq \bar{v}_{\frac{3}{2}}^{n+1}-\bar{v}_{\frac{1}{2}}^{n+1}=\bar{\delta}_{1} .
$$

Therefore, we conclude that

$$
\left(\delta_{0}\right)_{+}^{2}+\left(\delta_{1}\right)_{+}^{2}+\left(\delta_{2}\right)_{+}^{2} \leq\left(\bar{\delta}_{0}\right)^{2}+\left(\bar{\delta}_{1}\right)^{2}+\left(\bar{\delta}_{2}\right)^{2} .
$$

Case 4. $m=1$.

We already have $\left(\delta_{0}\right)_{+}^{2} \leq\left(\bar{\delta}_{0}\right)^{2}$. We still need to show that $\left(\delta_{1}\right)_{+}^{2} \leq\left(\bar{\delta}_{1}\right)^{2}$. If $\delta_{1} \leq 0$, then we are done. So suppose $\delta_{1}>0$ and observe that

$$
\delta_{1}=v_{\frac{3}{2}}^{n+1}-v_{\frac{1}{2}}^{n+1} \leq v_{1}^{n+1}-v_{\frac{1}{2}}^{n+1}=\bar{v}_{\frac{3}{2}}^{n+1}-v_{\frac{1}{2}}^{n+1} \leq \bar{v}_{\frac{3}{2}}^{n+1}-\bar{v}_{\frac{1}{2}}^{n+1}=\bar{\delta}_{1},
$$

and hence,

$$
\left(\delta_{0}\right)_{+}^{2}+\left(\delta_{1}\right)_{+}^{2} \leq\left(\bar{\delta}_{0}\right)^{2}+\left(\bar{\delta}_{1}\right)^{2} .
$$

Therefore, in all four cases $(m=1,2,3,4)$, we have

$$
\sum_{j=0}^{m}\left(\delta_{j}\right)_{+}^{2} \leq \sum_{j=0}^{m}\left(\bar{\delta}_{j}\right)^{2} .
$$

Now, we restrict the choice of $\sigma$ in the modified minmod limiter (see (2.10) ), to be like the one in MAPR (see (2.11)). Namely, we define $\mathrm{m}(a, b)$ in (2.10) with $\sigma$ such that

$$
\operatorname{sg}(\sigma)=\operatorname{sg}(s), \quad \text { where } \quad s= \begin{cases}a, & |a| \leq|b| \\ b, & |b| \leq|a| .\end{cases}
$$

Under this assumption on the limiter, we have the following result.

Theorem 3.4. Let $u^{0} \in L^{\infty}(\mathbb{R}), f$ be strictly convex in $R\left(u^{0}\right)$ and $f^{\prime \prime}$ be bounded on $\mathbb{R}$. Then there exists constant $\beta$ which depends only on the ratio $\gamma_{1} / \gamma_{2}$ and an absolute constant $c, c \geq \frac{1}{9000}$, such that under the CFL condition

$$
\lambda \max _{w \in R\left(u^{0}\right)}\left|f^{\prime}(w)\right| \leq \beta,
$$

the NT scheme with the minmod limiter $m(a, b)$ defined as in (2.10)-(3.34) satisfies the one-sided Lipschitz condition (3.4) provided that

$$
-\min \left(1, \frac{c}{2} \frac{\max \left(a_{+}, b_{+}\right)}{\min (|a|,|b|)}\right) \leq \sigma \leq 1 .
$$


Proof. The case $\sigma \geq 0$ follows from Theorem 3.1. Hence, we only consider the case $\sigma \leq 0$. This implies that we take a negative slope reconstruction at local minima and local maxima. Following the same steps as in the proof of Theorem 3.1, in the general case $(m \geq 4)$ we get

$$
\delta_{1}-\bar{\delta}_{1} \leq-\frac{1}{4} v_{0}^{\prime}, \quad \text { and } \quad \delta_{0}-\bar{\delta}_{0} \leq 0,
$$

where we recall that $\delta_{-\frac{1}{2}}<0 \leq \delta_{\frac{1}{2}}$ and $\sigma<0$ implies that $\left|\delta_{-\frac{1}{2}}\right| \leq \delta_{\frac{1}{2}}$. Thus,

$$
\left(\delta_{0}\right)_{+}^{2}+\left(\delta_{1}\right)_{+}^{2} \leq\left(\bar{\delta}_{0}\right)^{2}+\left(\bar{\delta}_{1}+\frac{1}{4}|\sigma| d\right)^{2},
$$

where $d=\min \left(\left|\delta_{-\frac{1}{2}}\right|,\left|\delta_{\frac{1}{2}}\right|\right)$. Notice that by (3.35), we obtain

$$
\frac{1}{2} \bar{\delta}_{1}|\sigma| d+\frac{1}{16}|\sigma|^{2} d^{2} \leq c\left(\frac{5}{16}\left(\delta_{\frac{1}{2}}\right)^{2}+\frac{1}{4} \delta_{\frac{1}{2}} \delta_{\frac{3}{2}}\right) \leq c\left(\left(\delta_{\frac{1}{2}}\right)^{2}+\left(\delta_{\frac{3}{2}}-2 \delta_{\frac{1}{2}}\right)^{2}\right),
$$

which implies

$$
\left(\delta_{0}\right)_{+}^{2}+\left(\delta_{1}\right)_{+}^{2} \leq\left(\bar{\delta}_{0}\right)^{2}+\left(\bar{\delta}_{1}\right)^{2}+c\left(\left(\Delta^{2} \bar{\delta}_{-\frac{1}{2}}\right)^{2}+\left(\Delta^{2} \bar{\delta}_{\frac{1}{2}}\right)^{2}\right) .
$$

By symmetric arguments, it can also be shown that

$$
\left(\delta_{m-1}\right)_{+}^{2}+\left(\delta_{m}\right)_{+}^{2} \leq\left(\bar{\delta}_{m-1}\right)^{2}+\left(\bar{\delta}_{m}\right)^{2}+c\left(\left(\Delta^{2} \bar{\delta}_{m-\frac{1}{2}}\right)^{2}+\left(\Delta^{2} \bar{\delta}_{m+\frac{1}{2}}\right)^{2}\right) .
$$

The remaining cases, $1 \leq m \leq 3$, can be handled in a similar way and we skip their proofs. We conclude that

$$
\sum_{j=0}^{m}\left(\delta_{j}\right)_{+}^{2} \leq \sum_{j=0}^{m}\left(\bar{\delta}_{j}\right)^{2}+c \sum_{j}\left(\Delta^{2} \bar{\delta}_{j+\frac{1}{2}}\right)^{2} \leq \sum_{j=0}^{m-1}\left(\delta_{j+\frac{1}{2}}\right)^{2},
$$

where the last inequality follows from the one-sided stability result proven in 10 for any nonnegative jump sequence; see (56) on page 553 in [10].

Remark 3.5. If the local jumps $a, b$ in the minmod limiter (2.10)-(3.34) are such that

$$
\frac{c}{2} \frac{\max \left(a_{+}, b_{+}\right)}{\min (|a|,|b|)} \geq 1
$$

we can recover the MAPR limiter taking $\sigma$ as in (2.11). Note that (3.42) is always true if $c \geq 2$. However, even though the bound $c \geq \frac{1}{9000}$ can be improved, the current approach does not allow us to prove the one-sided Lipschitz condition (3.4) with $c \geq 2$. Therefore, the minmod limiter (2.10)-(3.34) is more restrictive than MAPR in some cases.

Analogous to [10, a maximum principle and a one-sided stability result implies a convergence result. To state the convergence theorem we briefly introduce the space of functions of bounded variation and one-sided Lipschitz classes which are used in the context of conservation laws.

Definition 3.6. The space $\operatorname{Lip}\left(1, L^{1}(\mathbb{R})\right)$ consists of all functions $g \in L^{1}(\mathbb{R})$ such that the seminorm

$$
|g|_{\operatorname{Lip}\left(1, \mathrm{~L}^{1}(\mathbb{R})\right)}:=\limsup _{y>0} \frac{1}{y} \int_{\mathbb{R}}|g(x+y)-g(x)| d x
$$

is finite. 
For functions $g \in \operatorname{Lip}\left(1, \mathrm{~L}^{1}(\mathbb{R})\right)$ we consider the classes $\operatorname{Lip}\left(\mathrm{s}, \mathrm{L}^{\mathrm{p}}(\mathbb{R})\right)+$ defined by

$$
\left\|(g(\cdot-y)-g(\cdot))_{+}\right\|_{L^{p}(\mathbb{R})} \leq M y^{s}, \quad y>0 .
$$

The smallest $M \geq 0$ for which (3.44) holds is denoted by $|g|_{\operatorname{Lip}\left(\mathrm{s}, \mathrm{L}^{\mathrm{p}}(\mathbb{R})\right)+}$. When we set $p=\infty$ and $s=1$, we obtain the class $\operatorname{Lip}\left(1, L^{\infty}(\mathbb{R})\right)+$, which is the usual onesided Lipschitz class used in conservation laws denoted by Lip+; see for example [8. With this notation, by repeating exactly the same steps as in section 4 in [10, we obtain the following convergence result.

Theorem 3.7. Let $u^{0} \in \operatorname{Lip}\left(1, \mathrm{~L}^{1}(\mathbb{R})\right) \cap \operatorname{Lip}\left(1, \mathrm{~L}^{2}(\mathbb{R})\right)+$. Then, there exists $\beta>0$ such that under the CFL condition $\lambda\left\|f^{\prime}\right\|_{L^{\infty}(\mathbb{R})} \leq \beta$ the NT scheme based on the limiter (2.10), (3.34)-(3.35) converges to the unique entropy solution of (1.1).

\section{REFERENCES}

[1] Paul Arminjon and Marie-Claude Viallon. Généralisation du schéma de Nessyahu-Tadmor pour une équation hyperbolique à deux dimensions d'espace. C. R. Acad. Sci. Paris Sér. I Math., 320(1):85-88, 1995. MR.1320837 (95j:65115)

[2] Ivan Christov and Bojan Popov. New non-oscillatory central schemes on unstructured triangulations for hyperbolic systems of conservation laws. J. Comput. Phys., 227(11):5736-5757, 2008. MR2414928 (2009d:65102)

[3] Ami Harten, Björn Engquist, Stanley Osher, and Sukumar R. Chakravarthy. Uniformly highorder accurate essentially nonoscillatory schemes. III. J. Comput. Phys., 71(2):231-303, 1987. MR897244 (90a:65199)

[4] Ami Harten and Stanley Osher. Uniformly high-order accurate nonoscillatory schemes. I. SIAM J. Numer. Anal., 24(2):279-309, 1987. MR.881365 (90a:65198)

[5] G.-S. Jiang, D. Levy, C.-T. Lin, S. Osher, and E. Tadmor. High-resolution nonoscillatory central schemes with nonstaggered grids for hyperbolic conservation laws. SIAM J. Numer. Anal., 35(6):2147-2168 (electronic), 1998. MR1655841(99j:65145)

[6] Guang-Shan Jiang and Eitan Tadmor. Nonoscillatory central schemes for multidimensional hyperbolic conservation laws. SIAM J. Sci. Comput., 19(6):1892-1917 (electronic), 1998. MR:1638064 (99f:65128)

[7] Alexander Kurganov and Eitan Tadmor. New high-resolution central schemes for nonlinear conservation laws and convection-diffusion equations. J. Comput. Phys., 160(1):241-282, 2000. MR1756766 (2001d:65135)

[8] Haim Nessyahu and Eitan Tadmor. Nonoscillatory central differencing for hyperbolic conservation laws. J. Comput. Phys., 87(2):408-463, 1990. MR1047564(91i:65157)

[9] Stanley Osher and Eitan Tadmor. On the convergence of difference approximations to scalar conservation laws. Math. Comp., 50(181):19-51, 1988. MR917817 (89m:65086)

[10] Bojan Popov and Ognian Trifonov. One-sided stability and convergence of the NessyahuTadmor scheme. Numer. Math., 104(4):539-559, 2006. MR2249677(2008c:65221)

Department of Mathematics, Texas A\&M University, 3368 Tamu, College Station, TEXAS 77843

E-mail address: omehmet@math.tamu.edu

Department of Mathematics, Texas A\&M University, 3368 Tamu, College Station, TEXAs 77843

E-mail address: popov@math.tamu.edu 